\section{References}

1. Engler JR, Frede A, Saunders VA, Zannettino AC, Hughes TP, White DL. Chronic myeloid leukemia CD34+ cells have reduced uptake of imatinib due to low OCT-1 activity. Leukemia. 2010;24(4):765-770.

2. Carter BZ, Mak PY, Mu H, et al. Combined inhibition of MDM2 and $\mathrm{Bcr}-\mathrm{Abl}$ tyrosine kinase targets chronic myeloid leukemia stem/progenitor cells in a murine model. Haematologica. 2020;105(5):12741284.

3. Carter BZ, Mak PY, Mu H, et al. Combined targeting of BCL-2 and BCR-ABL tyrosine kinase eradicates chronic myeloid leukemia stem cells. Sci Transl Med. 2016;8(355):355ra117.

4. Lau LM, Nugent JK, Zhao X, Irwin MS. HDM2 antagonist Nutlin-3 disrupts p73-HDM2 binding and enhances p73 function. Oncogene. 2008;27(7):997-1003.

5. Willis SN, Chen L, Dewson G, et al. Proapoptotic Bak is sequestered by $\mathrm{Mcl}-1$ and $\mathrm{Bcl}-\mathrm{xL}$, but not Bcl-2, until displaced by $\mathrm{BH} 3$-only proteins. Genes Dev. 2005;19(11):1294-1305

6. Yoshimoto G, Miyamoto T, Jabbarzadeh-Tabrizi S, et al. FLT3-ITD up-regulates MCL-1 to promote survival of stem cells in acute myeloid leukemia via FLT3-ITD-specific STAT5 activation. Blood. 2009;114(24):5034-5043.

7. Hunter AM, Sallman DA. Current status and new treatment approaches in TP53 mutated AML. Best Pract Res Clin Haematol. 2019;32(2):134-144.

8. Carter BZ, Mak PY, Mak DH, et al. Synergistic effects of p53 activation via MDM2 inhibition in combination with inhibition of $\mathrm{Bcl}-2$ or $\mathrm{Bcr}-\mathrm{Abl}$ in CD34+ proliferating and quiescent chronic myeloid leukemia blast crisis cells. Oncotarget. 2015;6(31):30487-30499.

9. Inoue A, Kobayashi CI, Shinohara H, et al. Chronic myeloid leukemia stem cells and molecular target therapies for overcoming resistance and disease persistence. Int J Hematol. 2018;108(4):365370 .

10. Peterson LF, Mitrikeska E, Giannola D, et al. p53 stabilization induces apoptosis in chronic myeloid leukemia blast crisis cells. Leukemia. 2011;25(5):761-769.

11. Pavlovsky C, Chan O, Talati C, Pinilla-Ibarz J. Ponatinib in the treatment of chronic myeloid leukemia and philadelphia chromosome positive acute lymphoblastic leukemia. Future Oncol. 2019;15(3): 257-269.

12. Novotny L, Al-Tannak NF, Hunakova L. Protein synthesis inhibitors of natural origin for CML therapy: semisynthetic homoharringtonine (Omacetaxine mepesuccinate). Neoplasma. 2016;63(4):495-503.

13. Mattes K, Gerritsen M, Folkerts H, et al. CD34+ acute myeloid leukemia cells with low levels of reactive oxygen species show increased expression of stemness-genes and can be targeted by the BCL2 inhibitor Venetoclax. Haematologica. 2019 Nov 14. [Epub ahead of print]

14. Wang Q, Hao S. A-1210477, a selective MCL-1 inhibitor, overcomes ABT-737 resistance in AML. Oncol Lett. 2019;18(5):5481-5489.

15. Allan EK, Holyoake TL, Craig AR, Jørgensen HG. Omacetaxine may have a role in chronic myeloid leukaemia eradication through downregulation of Mcl-1 and induction of apoptosis in stem/progenitor cells. Leukemia. 2011;25(6):985-994.

16. Hanahan D, Weinberg RA. Hallmarks of cancer: the next generation. Cell. 2011;144(5):646-674.

\title{
Role of Meningioma 1 for maintaining the transformed state in MLL-rearranged acute myeloid leukemia: potential for therapeutic intervention?
}

\author{
Juerg Schwaller \\ University Children's Hospital beider Basel (UKBB), Department of Biomedicine (DBM), University of Basel, Switzerland \\ E-mail: JUERG SCHWALLER - j.schwaller@unibas.ch
}

doi:10.3324/haematol.2019.246348

$\mathrm{M}$ eningioma 1 (MN1) was cloned from a balanced chromosomal translocation in a meningioma as open reading frame encoding for a protein of 1,319 amino acids containing several proline and histidine-rich domains, acting as a transcriptional activator necessary for normal development of the bones of the skull. ${ }^{1,2}$ Several studies found mutations or aberrant expression of $\mathrm{MN1}$ in various hematologic malignancies. Characterization of a $\mathrm{t}(12 ; 22)(\mathrm{p} 13 ; \mathrm{q} 11)$ chromosomal translocation associated with myeloproliferative disorders revealed a fusion between MN1 and the ETSfamily transcription factor ETV6 (a.k.a. TEL). ${ }^{3}$ A fusion of $\mathrm{MN1}$ to Friend leukemia virus integration 1 (FLI1) has been shown to be a rare transforming oncogene in acute megakaryoblastic leukemia (AMKL). ${ }^{4}$ Aberrant high expression of $\mathrm{MN1}$ was reported in acute myeloid leukemia (AML) with inv(16) leading to the core-binding factor fusion CBFB-MYH11. ${ }^{5}$ Clinical studies proposed that high MN1 transcript levels could be used as prognostic marker in cytogenetically-normal (CN) AML. ${ }^{6}$ Functional studies in mice demonstrated the oncogenic potential of aberrant MN1 expression. Retroviral MN1 overexpression in murine bone marrow (BM) hematopoietic stem and progenitor cells (HSPC) followed by transplantation rapidly induced a lethal AML in mice. ${ }^{7}$ Aberrant MN1 expression due to retroviral insertion was shown to act as collaborative oncogenic event in acute leukemia induction by the MLL-ENL or the MLL-AF9 fusion gene, respectively., ${ }^{8,9}$ More recent work suggested that gene expression programs associated with MN1-mediated transformation of hematopoietic cells are controlled by the H3K4 and H3K79 histone methyltransferases MLL1 and DOT1L, respectively. ${ }^{10}$ Collectively, these studies indicated that aberrant MN1 expression contributes to malignant transformation of hematopoietic cells towards AML; however, its role in the maintenance of the transformed state remained poorly understood.

In a study published in this issue of Haematologica, Sharma et al. functionally addressed the role of MN1 in the maintenance of AML induced by MLL fusion oncogenes. ${ }^{11}$ They used Crisper/CAS9 to ablate MN1 in several murine AML lines, including cells transformed by retroviral overexpression of the MLL-AF9 fusion (rMLL-AF9), and in human THP1 and MV4;11 AML cell lines carrying the MLL-AF9 and MLL-AF4 fusions, respectively. They found that inactivation of MN1 impaired the clonogenic activity and proliferation associated with impaired cell cycle progression, and increased differentiation and apoptosis of murine rMLL-AF9 AML cells. Loss of MN1 also 
A Mouse AML cells

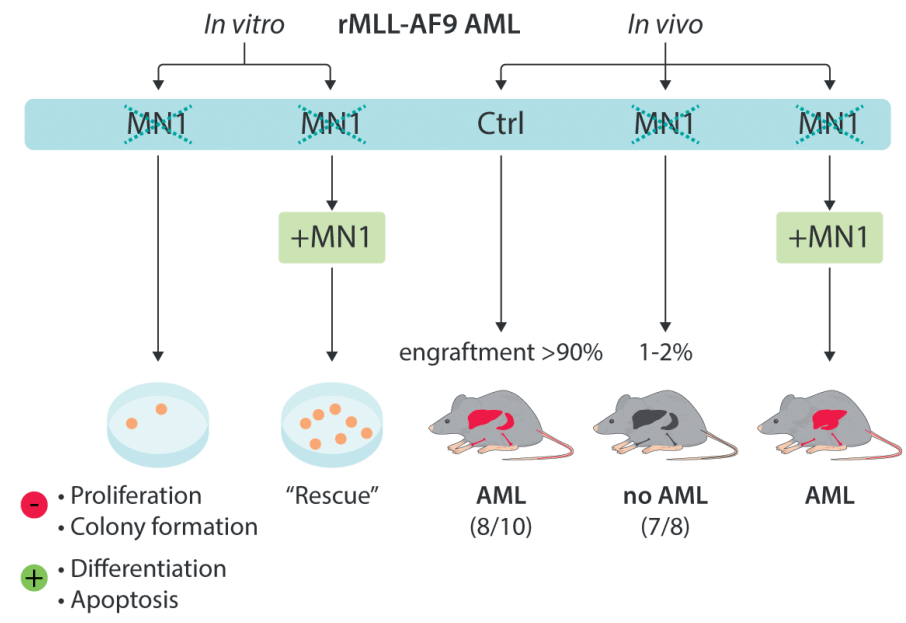

B Human AML cells

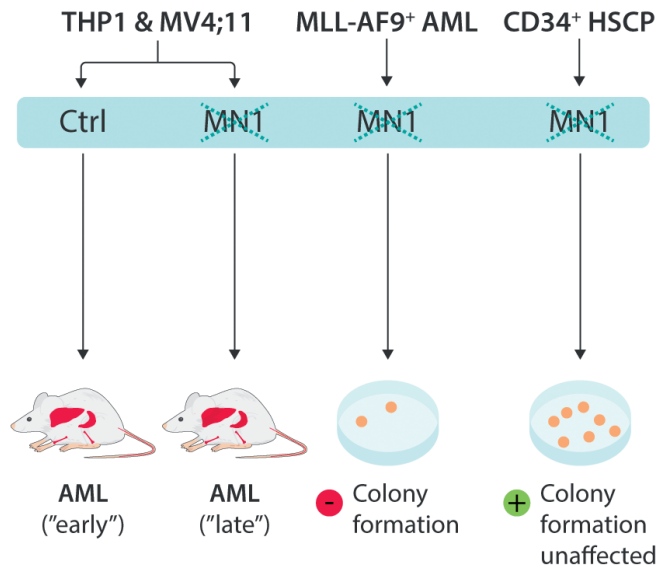

Figure 1. Schematic illustration of the findings by Sharma et al. in mouse (A) and human (B) acute myeloid leukemia (AML) cells expressing high levels of Meningioma 1 (MN1) and in $\mathrm{CD}_{34^{+}}$hematopoietic stem and progenitor cell (HSPC) controls (Ctrl).

reduced in vivo leukemia induction after transplanting the cells into syngenic mice. MLL-AF9+ AML cells lacking MN1 were significantly impaired in engrafting upon intravenous injection resulting in AML after long latency or no disease at all (Figure 1A). Notably, MN1 inactivation did not impair homing of transplanted cells to the BM. Most importantly, overexpression of exogenous MN1 'rescued' the anti-leukemic effects of ablation of endogenous MN1 in mouse rMLL-AF9 AML cells resulting in increased proliferation and clonogenic activity in vitro and increased engraftment and disease induction in vivo. Similar to murine cells, MN1 inactivation also delayed leukemia induction by transplantation of human THP1 or MV4;11 cells into immune deficient NSG mice. As in mouse rMLLAF9 AML cells, reduced MN1 expression (by siRNA) also impaired clonogenic activity of primary MLL-AF9 human AML cells, whereas colony formation by normal $\mathrm{CD} 34^{+}$ hematopoietic stem and progenitor cells (HSPC) was not affected (Figure 1B).

To understand the molecular mechanisms, Sharma et al. compared gene expression signatures of MLL-AF9+ leukemic cells before and after MN1 inactivation, and evaluated public chromatin immunoprecipitation sequencing (ChIP-seq) datasets. Enrichment of MN1 together with MLL-AF9, MEIS1 and the DOT1L-mediated H3K79me marks on the distal Hoxa gene cluster (Hoxa7Hoxa10) suggested that MN1 regulates Hoxa gene expression. However, MN1 seemed not to co-localize with MLLAF9, suggesting that MN1 primarily acts as a co-factor of Hoxa9 and Meis1. These observations were supported by reduced expression of the Hoxa9 target $B c l 2$ (and other Hoxa9/Hoxa10 targets) upon MN1 inactivation in MLL$\mathrm{AF9}^{+}$AML cells. Finally, Sharma et al. explored the effects of reduced MN1 levels in primary cells from five AML patients and in $\mathrm{CD} 34^{+}$HSCP from healthy donors. They observed that application of MN1-targeting siRNA, either packed in lipid nanoparticles or transfected, significantly reduced the number and size of colonies formed by primary MLL-AF9+ AML cells but had no effect on colony formation by normal HSPC in methylcellulose. Sharma et al. concluded that MN1 is essential to maintain a transformed state of AML cells expressing MLL-AF9. ${ }^{11}$

The experiments by Sharma et al. have been performed with well-characterized mouse and human MLL-AF9+ AML cells expressing high levels of MN1 and HOXA9. However, one has to keep in mind that not all AML cells carrying MLL-AF9 or other MLL rearrangements express aberrantly high levels of $\mathrm{MN1}{ }^{10}$ Although the effects of increased MN1 expression have been intensively studied, regulation of MN1 expression in hematopoietic cells still remains poorly understood. Previous reports found highest MN1 levels in hematopoietic stem cells (HSC) and early progenitor cells and very similar to HOXA genes, MN1 expression seems down-regulated during myelomonocytic differentiation. ${ }^{5,12}$ However, not all AML patients expressing high levels of MN1 also express high levels of the HOXA gene cluster. Inv(16)+ AML cells often associated with aberrantly high MN1 levels mostly express very low HOXA levels. ${ }^{13}$ Although Sharma et al. tested the efficacy of anti-MN1-siRNA in the inv $(16)^{+} \mathrm{ME1}$ cell line, they did not show whether MN1 knockdown impaired colony formation or proliferation of these cells as efficiently as in primary MLL-AF9+ murine AML cells.

Does MN1 expression reflect the cellular origin of transformation? Highest MN1 transcript levels were found in patients with immature $\mathrm{CD} 34^{+} \mathrm{AML} .{ }^{14}$ In addition, transformation by experimental MN1 overexpression was found to depend on activation of MEIS1/AbdB-like HOX proteins present in common myeloid progenitors (CMP) but not in more differentiated granulocyte-macrophage progenitors (GMP). ${ }^{15}$ Will the MN1 expression status change upon disease relapse? Experimental data indicated that overexpression of MN1 leads to resistance of AML cells to cytarabine and doxorubicin, suggesting selection for cells expressing highest levels during therapy. ${ }^{16}$ However, based on a small number of patients, there seemed to be only a slight trend towards higher MN1 mRNA expression upon disease relapse. ${ }^{17}$ Nevertheless, 
highest MN1 transcript levels were associated with a higher incidence and shorter time to relapse. ${ }^{14}$

How can we then therapeutically target MN1 expression? Although useful to provide proof of concept in experimental studies, clinical siRNA-based knockdown approaches, such as those used by Sharma et al., have so far been hampered by limited delivery into the target cells. However, small molecules have been generated that can interfere with certain transcription factor/co-factor protein-protein interactions or with transcription factor-DNA binding. In addition, transcription activity was successfully targeted by altering levels of ubiquitylation and subsequent proteasome degradation or by interference with regulators of transcription factor expression. ${ }^{18}$ To target MN1 as a transcription factor, we would need to know its potential interaction partners on chromatin and/or the critical domains of $\mathrm{MN1}$ that are necessary to maintain the transformed state of AML cells. Targeted genome editing screens could offer a platform to dissect structural needs of MN1 activity in AML cells. ${ }^{19}$ Previous work that explored the transforming potential of a large number of MN1 deletion mutants suggested that $221 \mathrm{~N}$-terminal amino acids are critical for induction of AML in vivo associated with expression of HOXA9, HOXA10 and MEIS2. ${ }^{20}$ Similarly, others reported that MN1 lacking amino acids 12-228 was unable to induce leukemia in the BM reconstitution assay, suggesting that overexpression of $\mathrm{N}$-terminal MN1 peptides and small molecules "mimicks" might be able to compete with potentially, yet to be defined, critical protein and/or chromatin interactions. ${ }^{21}$ Interestingly, the $\mathrm{N}$-terminal region of $\mathrm{MN1}$ was also shown to interact with the EP300 transcriptional co-activator, raising the question as to whether AML cells expressing high MN1 levels would be particularly sensitive to recently developed small molecule EP300 inhibitors. ${ }^{22,23}$

Collectively, by demonstrating a critical role for MN1 in AML maintenance, the work by Sharma et al. suggests that targeting the aberrantly high levels of MN1 expression would have strong anti-leukemic activity. However, the AML patients that would profit from such intervention, and the most efficient clinically applicable strategy, remain to be elucidated.

\section{Acknowledgments}

This work was supported by grants from the Swiss Cancer League (KFS-3487-08-2014) and the Swiss National Science Foundation (SNF, 31003_A_173224/1).

\section{References}

1. Lekanne Deprez RH, Riegman PH, Groen NA, et al. Cloning and characterization of $\mathrm{MN1}$, a gene from chromosome 22q11, which is disrupted by a balanced translocation in a meningioma. Oncogene. 1995;10(8):1521-1528.
2. Meester-Smoor MA, Vermeij M, van Helmond MJ, et al. Targeted disruption of the Mn1 oncogene results in severe defects in development of membranous bones of the cranial skeleton. Mol Cell Biol. 2005;25(10):4229-4236

3. Buijs A, Sherr S, van Baal S, et al. Translocation (12;22) (p13;q11) in myeloproliferative disorders results in fusion of the ETS-like TEL gene on $12 \mathrm{p} 13$ to the MN1 gene on 22q11. Oncogene. 1995;10(8):1511-1519.

4. Dang J, Nance S, Ma J, et al. AMKL chimeric transcription factors are potent inducers of leukemia. Leukemia. 2017;31(10):2228-2234.

5. Heuser M, Beutel G, Krauter J, et al. High meningioma 1 (MN1) expression as a predictor for poor outcome in acute myeloid leukemia with normal cytogenetics. Blood. 2006;108(12):3898-3905

6. Carella C, Bonten J, Sirma S, et al. MN1 overexpression is an important step in the development of inv(16) AML. Leukemia. 2007;21(8):1679-1690.

7. Heuser M, Argiropoulos B, Kuchenbauer F, et al. MN1 overexpression induces acute myeloid leukemia in mice and predicts ATRA resistance in patients with AML. Blood. 2007;110(5):1639-1647.

8. Liu T, Jankovic D, Brault L, et al. Functional characterization of high levels of meningioma 1 as collaborating oncogene in acute leukemia. Leukemia. 2010;24(3):601-612.

9. Bergerson RJ, Collier LS, Sarver AL, et al. An insertional mutagenesis screen identifies genes that cooperate with Mll-AF9 in a murine leukemogenesis model. Blood. 2012;119(19):4512-4523.

10. Riedel SS, Haladyna JN, Bezzant M, et al. MLL1 and DOT1L cooperate with meningioma-1 to induce acute myeloid leukemia. J Clin Invest. 2016;126(4):1438-1450.

11. Sharma A, Jyotsana N, Gabdoulline R, et al. Meningioma 1 is indispensable for mixed lineage leukemia-rearranged acute myeloid leukemia. Haematologica. 2020;105(5):1294-1305.

12. Kandilci A, Grosveld GC. Reintroduction of CEBPA in MN1-overexpressing hematopoietic cells prevents their hyperproliferation and restores myeloid differentiation. Blood. 2009;114(8):1596-1606.

13. Ichikawa H, Tanabe K, Mizushima H, et al. Common gene expression signatures in $\mathrm{t}(8 ; 21)$ - and inv(16)-acute myeloid leukaemia. $\mathrm{Br}$ J Haematol. 2006;135(3):336-347.

14. Jentzsch M, Bill M, Grimm J, et al. Prognostic Impact of Blood MN1 Copy Numbers Before Allogeneic Stem Cell Transplantation in Patients With Acute Myeloid Leukemia. HemaSphere. 2019;3(1): e167.

15. Heuser M, Yun H, Berg T, et al. Cell of origin in AML: susceptibility to MN1-induced transformation is regulated by the MEIS1/AbdBlike HOX protein complex. Cancer Cell. 2011;20(1):39-52.

16. Pardee TS. Overexpression of MN1 confers resistance to chemotherapy, accelerates leukemia onset, and suppresses p53 and Bim induction. PLoS One. 2012;7(8):e43185.

17. Carturan S, Petiti J, Rosso V, et al. Variable but consistent pattern of Meningioma 1 gene (MN1) expression in different genetic subsets of acute myelogenous leukaemia and its potential use as a marker for minimal residual disease detection. Oncotarget. 2016;7(45):7408274096.

18. Bushweller JH. Targeting transcription factors in cancer - from undruggable to reality. Nat Rev Cancer. 2019;19(11):611-624.

19. Shi J, Wang E, Milazzo JP, Wang Z, Kinney JB, Vakoc CR. Discovery of cancer drug targets by CRISPR-Cas9 screening of protein domains. Nat Biotechnol. 2015;33(6):661-667.

20. Lai CK, Moon Y, Kuchenbauer F, et al. Cell fate decisions in malig nant hematopoiesis: leukemia phenotype is determined by distinct functional domains of the MN1 oncogene. PLoS One. 2014;9 (11):e112671

21. Kandilci A, Surtel J, Janke L, Neale G, Terranova S, Grosveld GC. Mapping of MN1 sequences necessary for myeloid transformation. PLoS One. 2013;8(4):e61706.

22. van Wely KH, Molijn AC, Buijs A, et al. The MN1 oncoprotein synergizes with coactivators RAC3 and p300 in RAR-RXR-mediated transcription. Oncogene. 2003;22(5):699-709.

23. Lasko LM, Jakob CG, Edalji RP, et al. Discovery of a selective catalytic p300/CBP inhibitor that targets lineage-specific tumours. Nature. 2017;550(7674):128-132. 